Géraldine Barron et Pauline Le Goff-Janton, "Les ressources numériques, de quoi parle-t-on ? ", in Intégrer des ressources numériques dans les collections, Villeurbanne, Presses de l'enssib (La Boîte à outils 29), 2014, p. 14-20. https://books.openedition.org/pressesenssib/10856

\title{
Quelques définitions
}

\section{Les ressources numériques dans cet ouvrage}

La notion de "ressource numérique " convoque un certain nombre d'images fortement contrastées en fonction de la profession et de la culture du locuteur ; elle est aussi très marquée dans le temps. Car, littéralement, une ressource numérique est un ensemble de moyens représentés par des chiffres, exploitables grâce à l'outil informatique. Rien de plus vague qu'une pareille notion. Il serait d'ailleurs plus exact de parler d'information dématérialisée, même si la question du support de lecture reste importante. Il paraît évident aujourd'hui d'évacuer les supports de l'information numérique qui ont fait les beaux jours des bibliothèques des années 2000 (cédéroms, CD audio, DVD, etc.). II ne s'agit pas dans cette contribution de proposer un inventaire à la Prévert des différentes « ressources numériques " non plus que d'en faire une typologie mais plutôt de souligner les problématiques qui s'y rapportent pour les bibliothèques et centres de documentation, à partir de quelques choix typologiques forcément arbitraires et réducteurs du fait de leur fondamentale plasticité.

Pour un bibliothécaire, l'expression évoque, de façon première, de l'information bibliographique ou des contenus éditoriaux acquis, ce qui sous-entend :

- un producteur externe : éditeur, agrégateur, avec lequel la bibliothèque est engagée dans une relation commerciale ;

- une « ressource » basée sur le couple notice bibliographique - information/contenu.

Les " ressources numériques " regroupent ainsi les produits commerciaux proposant l'accès à un contenu en ligne, accessible aux usagers de la bibliothèque ${ }^{1}$, et pour la mise à disposition desquels une dépense d'acquisition inscrite dans le budget de la bibliothèque a été réalisée, quelles qu'en soient les modalités (abonnement à une base de données, achat de licences, achat pérenne...).

On y trouve, en vrac et sans prétendre à l'exhaustivité, des bases de données bibliographiques, des livres, des bouquets de journaux et revues, des méthodes d'apprentissage en ligne, de la musique, des vidéos, voire des jeux vidéo, autant de ressources dématérialisées qui renvoient à l'offre traditionnelle des bibliothèques. La différence majeure se situe dans le support - ou plutôt l'absence de support qui nécessite de déployer de nouvelles stratégies de signalement et de médiation ${ }^{2}$ et d'apprendre à gérer la masse d'information que permet cette dématérialisation. Cet ouvrage traite essentiellement de ces produits commerciaux, et de leur cortège de problématiques de sélection, de coût, d'accès, de signalement, de gestion et d'archivage, qui ne sont une fois encore que la transposition dans un " univers numérique » du circuit de traitement du document.

Bien qu'il ne soit plus question de support, l'accès à ces ressources est particulièrement hétérogène et source de désarroi pour le professionnel comme pour l'usager : fichiers téléchargeables ou non, avec ou sans système de gestion des droits (Digital Rights Management ou DRM)*, imprimables en totalité ou en partie, consultables uniquement via une interface propriétaire, plus ou moins confortable, plus ou moins ergonomique, plus ou moins portable, et qui peut réclamer en outre une installation logicielle

\footnotetext{
${ }^{1}$ Ce critère de l'accès du public à la ressource exclut notamment du champ des ressources numériques ici traitées les outils professionnels tels que la base Electre.

${ }^{2}$ Voir la partie III : « Intégrer, signaler », p. 89.
} 
spécifique (lecteurs PDF ou vidéo). Plus la bibliothèque offre de ressources différentes, plus leur manipulation est déconcertante et manque singulièrement d'unité et de repères.

Cette conception des ressources numériques est très réductrice, puisqu'elle met de côté les problématiques spécifiques aux ressources numériques produites par la bibliothèque ou l'institution dans laquelle celle-ci s'inscrit (collectivité ou université), ou encore aux ressources gratuites disponibles sur Internet. Les bibliothèques numériques agrègent en effet plusieurs types de ressources hétérogènes, dont l'intérêt ne se mesure pas à la dépense engagée par la bibliothèque pour les acquérir et dont la mise en cohérence relève de la politique de l'établissement. Envisager les ressources numériques dans cette acception large dépassait cependant le cadre de cet ouvrage, dont le volume ne permet pas de traiter dans le détail de toutes leurs déclinaisons. Se concentrer sur un type de ressource ne signifie cependant pas ignorer les autres, d'autant que les enjeux que suscitent les différents types se croisent bien souvent.

Quelques enjeux des ressources numériques situées en dehors du champ de cet ouvrage

Des ressources en accès gratuit

Le contenu du Web dans son ensemble constitue un immense réservoir d'information numérique, plus ou moins intéressante et pertinente, mais suffisamment importante dans son entièreté pour mériter le développement de pratiques spécifiques d'archivage et de consultation rétrospective ${ }^{3}$. Les pratiques traditionnelles de sélection et de signalement d'une information à destination d'un lectorat potentiel s'appliquent à l'information numérique présente sur le Web, même si cette information présente un caractère instable inconnu du temps des supports matériels. Les sites et pages Web font le plus souvent l'objet d'un signalement exogène au catalogue, peu dynamique (signets, listes) ou au contenu non maîtrisé (agrégateurs de flux). Quelle que soit la solution technique adoptée, il s'agit de pousser de l'information vers un usager qui peut y avoir accès directement, sans que l'intervention du bibliothécaire apporte une réelle valeur ajoutée. Cette problématique de sélection-signalementmédiation se retrouve, bien que dans une moindre mesure, pour la gestion des ressources éditoriales en accès gratuit ${ }^{4}$ (livres, revues libres de droit ou en libre accès, archives ouvertes) qui interroge la légitimité même du bibliothécaire ou du documentaliste : à partir du moment où un texte est librement accessible sur le Web, quel besoin y-a-t-il que ma bibliothèque me le propose ? Comment et sous quelle forme ? La réponse variera évidemment selon le type de bibliothèque et ses publics, mais elle renvoie fondamentalement à des problématiques de politique documentaire qui nécessitent une bonne connaissance de l'environnement documentaire, non plus de la bibliothèque sous l'angle géographique, mais du lectorat, des besoins et des attentes de ces publics ; à la différence des ressources matérielles ou même des ressources numériques payantes, il ne s'agit plus de définir des orientations budgétaires mais des capacités signalétiques : il faut gérer la masse d'information (l'infobésité), conserver la maîtrise de ce que l'on signale (capacité de traitement) et être en mesure de le valoriser (médiation numérique).

\footnotetext{
${ }^{3}$ Comme, par exemple, l'archivage du Web à la Bibliothèque nationale de France (BnF). [En ligne] : http://www.bnf.fr/fr/collections et services/livre presse medias/a.archives internet.html.

${ }^{4}$ Voir la contribution de Delphine Coudrin et Guillaume Hatt : « Intégrer, signaler les ressources électroniques : 20 questions- clés », p. 90.
} 


\section{La bibliothèque productrice de contenu et de données}

L'ensemble des opérations de sélection, traitement, signalement, valorisation-médiation des ressources numériques est lui-même générateur de nouveaux contenus numériques ${ }^{5}$ : la bibliothèque est donc elle aussi créatrice de ressources numériques, bibliothéconomiquement qualifiables (collections numérisées) ou non (sites Web, blogs, pages d'agrégation de flux, réseaux sociaux, services de questions-réponses, etc.), dans un effet de miroir qui peut se révéler vertigineux ou stérile. La bibliothèque n'est d'ailleurs pas seulement créatrice de contenus : elle est aussi productrice de données qu'elle peut choisir d'exposer, d'exploiter et de partager. Les notices bibliographiques et autres réservoirs de métadonnées constituent eux aussi des ressources numériques : on en veut pour preuve le fait que des sociétés commerciales proposent des banques de données bibliographiques. Gérer les ressources numériques, c'est aussi se poser la question de la propriété de ces données bibliographiques créées ou importées, modifiées, réappropriées. Sans aller jusqu'à la notion archivistique qui impliquerait que le bibliothécaire réfléchisse sur le devenir des emails, factures numériques et autres procédures administratives, il ne faut pas perdre de vue que le professionnel de I'information est aujourd'hui autant producteur qu'utilisateur et médiateur de ressources numériques. Le format des ouvrages de la Boîte à outils ne permet pas d'embrasser aussi large, et ce n'est d'ailleurs pas souhaitable car cela ferait perdre de vue l'aspect pratique fait de recommandations dont un responsable des ressources numériques nouvellement nommé a besoin pour se familiariser avec un environnement complexe, déstructuré mais néanmoins codifié. Différentes « ressources numériques " seront donc évoquées au gré des contributions, souvent plus dans le fil d'une réflexion théorique que de solutions pratiques, car le champ n'est pas encore balisé dans toute son étendue.

\section{Taux de pénétration et impact budgétaire}

Les ressources numériques payantes bénéficient pour les bibliothécaires et leurs tutelles d'un surcroît de visibilité dans des contextes budgétaires contraints.

Les travaux menés dans le cadre du Schéma numérique des bibliothèques ${ }^{6}$ montraient qu'en 2008, un tiers du budget documentaire des bibliothèques universitaires était déjà consacré à l'acquisition de la documentation électronique, soit un total de $\mathbf{3 0}$ millions d'euros. Ces chiffres sont en augmentation constante, et dans quelques cas extrêmes, l'achat de documentation papier tend à disparaître. Pour les bibliothèques universitaires, l'hybridation des collections constitue désormais un enjeu complexe sur le plan budgétaire comme en termes de publics à desservir. Si, selon l'Enquête ADBU 2002-2014: évolution des budgets d'acquisition des bibliothèques universitaires, 57,6\% des dépenses d'abonnements destinées aux laboratoires concernent désormais des ressources électroniques, l'offre éditoriale française destinée aux étudiants, notamment les manuels, demeure majoritairement imprimée. Le coût des ressources électroniques à destination des laboratoires s'étant accru de 457,6 \% entre 2002 et 2014, alors que les budgets documentaires n'ont pas augmenté dans les mêmes proportions $^{7}$ : " le choix des universités françaises a clairement été de maintenir les ressources électroniques à destination des laboratoires, quel qu'en soit le coût [...], au détriment des étudiants ${ }^{8} »$.

\footnotetext{
${ }^{5}$ Voir la BAO\#30 : Produire des contenus documentaires en ligne : quelles stratégies pour les bibliothèques ?, sous la direction de Christelle Di Pietro, octobre 2014.

${ }^{6}$ Voir le Schéma numérique des bibliothèques, remis par Bruno Racine le 22 mars 2010 au ministre de la Culture et de la Communication. [En ligne] : http://www.ladocumentationfrancaise.fr/rapports-publics/104000143/

${ }^{7}$ Les budgets documentaires des bibliothèques universitaires (BU) ont augmenté de 43,5\% entre 2002 et 2010 , avant de connaître une stagnation en 2011 et 2012 puis une diminution de 3,3 \% entre 2012 et 2014 .

${ }^{8}$ Enquête ADBU 2002-2014 : évolution des budgets d'acquisition des bibliothèques universitaires. [En ligne] : http://adbu.fr/wp-content/uploads/2014/03/Enqu\%C3\%AAte ADBU 2014.pdf
} 
Des études statistiques sont menées régulièrement par les établissements, mais aussi au niveau national par le consortium Couperin. Elles tendent à montrer que si les coûts continuent d'augmenter, les usages, qui ont connu des hausses continuelles et importantes pendant une dizaine d'années, ont aujourd'hui tendance à stagner voire à régresser, preuve que l'usage des produits, bien implantés dans le paysage documentaire de la recherche, est parvenu à un plafond.

La dématérialisation de l'information et des produits culturels a touché plus tardivement les bibliothèques territoriales. L'enquête menée par le Service du livre et de la lecture en partenariat avec l'association Réseau Carel fin 2013 sur les ressources numériques des bibliothèques publiques ${ }^{9}$ dresse un premier panorama chiffré de la situation. $23 \%$ des bibliothèques municipales (BM) desservant plus de 10000 habitants et $54 \%$ des bibliothèques départementales de prêt (BDP) proposent des ressources numériques en 2013, alors qu'elles n'étaient respectivement que $14 \%$ et $43 \%$ à le faire en 2011. La transition numérique des bibliothèques des grandes agglomérations est logiquement plus avancée, puisque $82 \%$ des BM desservant plus de 70000 habitants proposent des ressources numériques et que $50 \%$ d'entre elles dépensent plus de 30000 euros pour ces acquisitions, quand le budget moyen consacré aux ressources numériques par l'ensemble des BM enquêtées est d'environ 12700 euros. La part des acquisitions numériques dans le budget documentaire des bibliothèques territoriales est désormais d'environ $8 \%{ }^{10}$.

La typologie des ressources proposées par les bibliothèques territoriales est la suivante :

- 60 \% des BM proposant des ressources numériques proposent au moins une ressource d'autoformation ;

- $51 \%$ une ressource de presse ;

- $48 \%$ des livres numériques ${ }^{11}$;

- $40 \%$ des ressources audiovisuelles en ligne.

Ces ressources, dont les bibliothèques publiques déploraient la très faible utilisation depuis le milieu des années 2000, connaissent depuis deux ans une hausse significative de leurs usages, ainsi qu'une certaine fidélisation de leurs publics. Plusieurs facteurs permettent d'expliquer cette hausse : développement de l'offre, mise en place par les bibliothèques de dispositifs de médiation, possibilité pour l'usager d'accéder aux ressources sur ses appareils mobiles, etc. Seule l'offre de livres numériques apparaît aujourd'hui encore assez faible, comparativement à d'autres produits et à l'offre disponible pour les usagers des bibliothèques américaines.

Limiter le champ d'étude aux ressources payantes permet d'aborder un terrain aujourd'hui relativement bien balisé. Ce n'est pas encore le cas des ressources gratuites qui ouvrent une nouvelle frontière aux pratiques bibliothéconomiques qui ont su s'adapter (ou adapter l'offre) à un cadre qui leur était relativement familier en termes de signalement (métadonnées, Digital Object Identifier [DOI]), de localisation (URL pérenne), d'évaluation de I'usage (statistiques), de structure et de granularité*. Des solutions peuvent être apportées pour faciliter l'intégration de ressources gratuites

9 Enquête 2013-2014 sur les ressources numériques en bibliothèque publique. [En ligne] : http://www.culturecommunication.gouv.fr/content/download/89759/671923/file/Enquete ressources numer iques bibliotheques mars2014.pdf

${ }^{10} \mathrm{Si}$ ce pourcentage est loin du poids représenté par les ressources numériques dans le budget documentaire des $\mathrm{BU}$, la dépense des bibliothèques territoriales consacrée à l'acquisition de ressources numériques ne représentait environ que $2 \%$ de leur budget documentaire à l'époque du Schéma numérique des bibliothèques. ${ }^{11} 67 \%$ des BDP offrant des ressources numériques proposent des livres numériques, devant tous les autres types de ressources. 
dans l'offre documentaire des bibliothèques ${ }^{12}$, mais il s'agit d'une part de solutions payantes, d'autre part, de calquer un modèle sur un autre, alors que la majorité des infinies ressources du Web échappent à cette structuration héritée de l'édition traditionnelle. Puisqu'il n'est pas encore possible de proposer des conseils pratiques à l'heure où les établissements tâtonnent, nous nous contenterons de proposer, lorsque c'est possible, un focus sur ces " autres ressources " qui restent en filigrane de l'ouvrage et des préoccupations des professionnels.

${ }^{12}$ Citons en particulier le modèle Freemium* adopté par OpenEdition qui a ouvert la voie à de nouvelles formes de financement et de valorisation des ressources en libre accès par la mise en place de services payants dédiés aux bibliothèques. 